\title{
Basic fibroblast growth factors as a biomarker of focal segmental glomerulosclerosis in HIV-positive and HIV-negative children
}

\author{
N Z Gumede, ${ }^{1}$ MB ChB, MMed Sci; T Naicker, ${ }^{2} \mathrm{PhD} ; \mathbf{R}$ Bhimma, ${ }^{1} \mathrm{MB} \mathrm{ChB}, \mathrm{PhD}$ \\ ${ }^{1}$ Department of Clinical Medicine, Nelson R. Mandela School of Medicine, College of Health Sciences, University of KwaZulu-Natal, Durban, \\ South Africa \\ ${ }^{2}$ Department of Paediatrics and Child Health, Nelson R. Mandela School of Medicine, College of Health Sciences, University of KwaZulu-Natal, \\ Durban, South Africa
}

Corresponding author: NZ Gumede (nokwandah.gumedeh@gmail.com)

\begin{abstract}
Background. HIV infection can lead to the development of HIV-associated nephropathy (HIVAN) with the majority of patients progressing to end-stage kidney disease. Previous studies have recognised basic fibroblast growth factor (bFGF) as a biomarker for HIVAN, since significant levels of bFGF low-affinity receptors have been found in the kidneys of HIV-infected children.

Objective. To assess the association between bFGF and kidney disease in the development of focal segmental glomerulosclerosis (FSGS) in HIV-positive and negative children.

Methods. The study group consisted of 31 children; HIVAN $(n=11)$ and idiopathic FSGS $(n=20)$. The control group consisted of both HIV-positive $(n=20)$ and HIV-negative $(n=20)$ children with no kidney disease. Serum samples from all patients in both the study and control groups were analysed for bFGF.

Results. The concentration of bFGF was higher, in comparison with idiopathic FSGS children, in HIVAN children $(p=0.0167)$. There was also a significant elevation of serum bFGF levels in children with HIVAN when compared with HIV-positive $(p=0.0288)$ and HIV-negative $(p=0.0043)$ control groups.

Conclusion. This study demonstrated statistically significant differences between bFGF levels in children with HIVAN and a control group, although it failed to distinguish significant differences in bFGF levels between HIVAN and idiopathic FSGS children.
\end{abstract}

S Afr J Child Health 2020;14(2):62-65. https://doi.org/10.7196/SAJCH.2020.v14i2.1589

HIV-infected antiretroviral therapy (ART) naive children display a high viral load during the late phase of infection, placing them at risk of developing several types of kidney diseases, including HIV-associated nephropathy (HIVAN) ${ }^{[1]}$ However, kidney disease is usually uncommon in children receiving ART. ${ }^{[2]}$ The spectrum of kidney disease that occurs with HIV infection includes acute kidney injury, disorders of tubular function, thrombotic microangiopathies, kidney injury secondary to drug use and various forms of chronic glomerular diseases (including HIVAN and HIV-associated immune complex disorders). ${ }^{[3]}$ The classical findings of HIVAN include persistent proteinuria that is usually accompanied by varying degrees of haematuria, urinary sediment with urinary microcysts, azotaemia, normal to large kidneys on ultrasound images, normal blood pressure and focal segmental glomerulosclerosis (FSGS) on kidney biopsy findings. ${ }^{[2,4]}$

FSGS is an important comorbidity in HIV infection. ${ }^{[5]}$ FSGS is a primary cause of nephrotic syndrome in children and, if untreated, has a poor prognosis. ${ }^{[6]}$ Nonetheless, the pattern of segmental and focal sclerosis is not specific to disease with primary podocyte lesions, and several other diseases e.g. HIVAN demonstrate light microscopic lesion patterns such as overlying cell hyperplasia as well as glomerular tuft injuries similar to primary FSGS. ${ }^{[7]}$ In the pre-ART era, HIVAN was characterised by rapid progression to end-stage kidney disease leading to the need for dialysis. ${ }^{[8]}$ Highly active ART (HAART) has improved the natural course of this disease, increasing the importance of early diagnosis and allied suitable care. ${ }^{[8]}$ Robust biomarkers may assist in diagnosing and monitoring kidney disease commencing from initial stages of the disease. ${ }^{[9]}$
Previous studies have recognised basic fibroblast growth factor (bFGF) as a biomarker for FSGS, as significant levels of bFGF lowaffinity receptors have been found in the kidney of HIV-infected children. ${ }^{[10]}$ bFGF is an angiogenic vascular growth factor that is essential for cellular aggregation induction. ${ }^{[11]}$ It is involved in the stimulation of epithelial condensation, apoptosis inhibition as well as maintenance of WT1 synthesis. ${ }^{[12]}$ Furthermore, bFGF is mainly stored as an inactive pool within the extracellular matrix and the vessel wall, and is absent in the circulation unless it is secreted during tissue injury and angiogenesis. ${ }^{[13]}$ Elevated levels of bFGF may result in the progression of FSGS, and unrestrained elevation of bFGF in the kidney extracellular matrix can generate the fibrotic lesions and tubulointerstitial proliferation distinctive to HIVAN. ${ }^{[13]}$ However, the processes that regulate secretion and behaviour of bFGF in glomeruli and kidney tubules are as yet not clearly understood. ${ }^{[14]}$ Based on the findings that elevated bFGF may result in the progression of FSGS and plays a role in the generation of fibrotic lesions and tubulintersitial proliferation distinct to HIVAN, our aim was to assess the association between bFGF and kidney disease in the development of FSGS in HIV-positive and HIV-negative children.

\section{Objective}

To investigate the development of FSGS in HIV-positive and HIVnegative children.

\section{Methods}

Blood samples from black South African (SA) children aged between 
1 and 16 years, from KwaZulu-Natal Province, with biopsy-proven HIVAN $(n=11)$ and idiopathic FSGS $(n=20)$ were employed in this study. A control group $(n=40)$ was further stratified into HIVpositive children with no kidney disease $(n=20)$ and HIV-negative children with no kidney disease $(n=20)$. Ethical approval was received from the University of KwaZulu-Natal Biomedical Research Ethics Committee (ref. no. 256/12) and permission to perform the study was given by the respective managers of Inkosi Albert Luthuli Central and King Edward VIII hospitals. Furthermore, written informed consent was obtained from the children's parents or legal guardian prior to collection of blood samples. All identifiers were removed and, following collection, each blood sample was assigned a unique study identification number. All data collected were stored on a password-protected spreadsheet.

All patients were stable on HAART for over 6 months before being recruited. Estimated glomerular filtration rate (eGFR) was calculated using the modified Swartz formula. ${ }^{[15]}$

Serum was obtained and stored at $-80^{\circ} \mathrm{C}$, before bFGF levels were quantified, as mean fluorescence intensity (MFI), using the Bio-Plex Pro Human Cytokine assay (Bio-Rad laboratories Inc., USA) on a Bio-Plex MAGPIX Multiplex system (Bio-Rad Laboratories Inc., USA) using Bio-Plex Manager version 4.1 software.

Data were entered into SPSS version 24 (IBM Corp., USA) and GraphPad Prism version 5 (GraphPad Software, USA) for analysis. A $p$-value $<0.05$ was considered as statistically significant. A descriptive statistical analysis of the data was conducted prior to inferential analysis. Independent samples $t$-test and ANOVA were used to determine if high levels of bFGFs in FSGS compared with controls were responsible for the development of glomerulosclerosis. $\chi^{2}$ tests were used to assess any associations between categorical variables.

\section{Results}

The overall study population comprised 71 children, 31 of whom had a histopathological pattern of FSGS (Table 1). The mean (SD) age for idiopathic FSGS and HIVAN children was 9 (3.1) and 10 (3.6) years, respectively.

To evaluate the association between bFGF and kidney disease, bFGF concentration was compared with age, weight and laboratory findings that included blood urea, serum creatinine, eGFR and serum cholesterol in all participants, and CD4 cell counts in HIV-positive participants (Table 1). Non-significant statistical correlations were demonstrated, therefore signifying that these demographic data had no observable effect on the bFGF MFI in children.

There was a significant elevation of serum bFGF levels in the children with HIVAN when compared with the HIV-positive and HIV-negative control groups (Table 1). There was no statistically significant difference in bFGF concentration between children with idiopathic FSGS and those within the positive and negative control groups.

\section{Discussion}

Several studies have confirmed the presence of HIVAN in children who are most likely to develop nephrotic syndrome in association with FSGS. ${ }^{[10]}$ This study reports a significant elevation of serum bFGF levels in children with HIVAN when compared with the HIV-positive and HIV-negative control groups. Similarly a study by Liu et al.$^{[13]}$ detected elevated levels of bFGF in HIVAN children. HIVAN is associated with an increased expression of kidney heparan sulphate proteoglycans that may facilitate the accumulation of bFGF in the kidney and the progression to end-stage kidney disease. Furthermore, kidney tubular epithelial cells harvested from the serum of children with HIVAN produce and release high levels of bFGF, as well as a FGF-binding protein, that facilitates the release of several members of the FGF's family, including bFGF. ${ }^{[13]}$ These findings may explain the elevated levels of serum bFGF in children with HIVAN. However, our study observed no significant difference in bFGF levels between HIVAN and idiopathic FSGS children.

We noted elevated albumin levels in HIVAN children. These results corroborate findings that increased levels of albumin result from improved appetite and general well-being as well as decreased loss of protein in the urine. ${ }^{[14]}$ Proteinuria is a marker of HIV nephropathy and has been correlated with decreased kidney function and progression to end-stage renal disease. ${ }^{[15]}$ It is important to note that in the present study, all HIV-infected children were on HAART. HAART, and angiotensin-converting enzyme antagonists are highly effective in reducing proteinuria and protecting kidney function. ${ }^{[16]}$

In this study, although not statistically significant, higher levels of creatinine were measured in children with HIVAN compared with children with idiopathic FSGS. This finding could influence higher levels of bFGF in children with HIVAN as it has been shown that plasma FGF23 concentration increases progressively as glomerular fuiltration rate declines. ${ }^{[17]}$ These parameters further

Table 1. Demographic data and laboratory findings of patients

\begin{tabular}{|c|c|c|c|c|}
\hline & \multicolumn{4}{|c|}{ mean (SD) } \\
\hline & $\begin{array}{l}\text { HIVAN } \\
(n=11)\end{array}$ & $\begin{array}{l}\text { Idiopathic FSGS } \\
(n=20)\end{array}$ & $\begin{array}{l}\text { HIV-positive control } \\
(n=20)\end{array}$ & $\begin{array}{l}\text { HIV-negative control } \\
(n=20)\end{array}$ \\
\hline Age (years) & $10(3.6)$ & $9(3.1)$ & $11(3.2)$ & $7(3.9)$ \\
\hline Weight (kg) & $31.13(11.57)$ & $29.04(10.33)$ & $37.25(13.73)$ & $18.20(10.55)$ \\
\hline Serum creatinine $(\mu \mathrm{mol} / \mathrm{L})$ & $82(62)$ & $45(15)$ & $40(9)$ & $29(9)$ \\
\hline Urine protein $(\mathrm{g} / \mathrm{L})$ & $3.07(2.04)$ & $4.22(4.21)$ & - & $1.85(0.67)$ \\
\hline eGFR (mL/min/1.73sqm) & $119(57)$ & $125(102)$ & $207(58)$ & $218(122)$ \\
\hline Blood urea (mmol/L) & $5.30(4.26)$ & $7.64(7.02)$ & $5.34(9.81)$ & $2.71(1.58)$ \\
\hline Albumin $(\mathrm{g} / \mathrm{L})$ & $29.91(14.76)$ & $28.96(7.96)$ & $30.77(10.94)$ & $37.30(9.25)$ \\
\hline Cholesterol (mmol/L) & $4.82(2.36)$ & $8.21(4.80)$ & $3.75(0.68)$ & - \\
\hline CD4 count & $820(642)$ & $900(620)$ & - & - \\
\hline bFGF $(\mathrm{ng} / \mathrm{mL})^{*}$ & $8.682(10.18-7.185)^{\dagger}$ & $7.375(8.16-6.59)$ & $6.975(7.65-6.385)^{\dagger}$ & $6.538(7.006-6.069)^{\dagger}$ \\
\hline \multicolumn{5}{|c|}{$\begin{array}{l}\mathrm{SD}=\text { standard deviation; FSGS }=\text { focal segmental glomerulosclerosis; HIVAN }=\text { HIV-associated nephropathy; HIV }=\text { human immunodeficiency virus; bFGF }=\text { basic fibroblast } \\
\text { growth factor. } \\
{ }^{*} \text { Mean }(95 \% \text { confidence interval). } \\
\text { 'Denotes a statistically significant difference, based upon the Mann-Whitney } U \text { test, between HIVAN group and both the HIV-positive }(U=58.8 ; p=0.0288) \text { and HIV-negative } \\
(U=43.5 ; p=0.0043) \text { control groups. }\end{array}$} \\
\hline
\end{tabular}


support the view that children with HIV-associated FSGS treated with HAART may achieve full remission and that the treatment may delay or prevent progression to end-stage kidney disease. Such findings are supported by a study on 152 biopsy-proven HIVAN patients treated with HAART who were found to have better kidney survival compared with patients who did not receive HAART. ${ }^{[18]}$ Hence, HIVAN should be considered as an indication to initiate HAART. HIVAN is common in patients who are newly diagnosed with advanced HIV infection or those who are no longer undergoing antiviral therapy ${ }^{[19]}$ It is reported that a well-controlled HIV infection and continued antiviral therapy have a substantial influence on HIVrelated kidney disease delaying rapid progression to ESRD. ${ }^{[19]}$

In this study, the bioactive protein bFGF was significantly lower in HIV-negative and HIV-positive control groups compared with HIVAN children. Possible reasons for the significantly elevated bFGF level in children with HIVAN include stimulation of cell growth by bFGF. ${ }^{[20]}$ High levels of bFGF may lead to the development of FSGS. ${ }^{[13]}$ bFGF can increase the attachment of HIV-infected mononuclear cells to kidney epithelial cells, ${ }^{[19]}$ enhance the expression of hypoxiainducible genes in these cells, ${ }^{[15]}$ induce kidney microcysts, ${ }^{[21]}$ and/ or cause FSGS. ${ }^{[22]}$ Excessive accumulation of bFGF in the kidney extracellular matrix can induce tubulointerstitial proliferative and fibrotic lesions typical of HIVAN. ${ }^{[13]}$ However, the mechanisms that control the release and activity of bFGF in kidney tubules and glomeruli are not clearly described.

The present study was based on previous investigations that reported an accumulation of heparin-binding growth factors in association with the occurrence of biomarkers for children with HIVAN, namely kidney microcytic tubular lesions. ${ }^{[1]}$ Another study based on this approach, revealed significant differences in the levels of bFGF between HIVAN and a control group, with bFGF elevated in HIVAN. In support of the latter finding, our study also reports similar results.

In this study, no significant difference was observed between idiopathic FSGS and, HIV-positive and HIV-negative control groups as well as between children having idiopathic FSGS or HIVAN. This finding may be attributed to the small sample size available for use in this study and/or that the patients had established disease and were already on treatment, with arrested or markedly attenuated distal tubular cell injury.

The present study, and that of Wyatt, ${ }^{[20]}$ demonstrate that bFGF has potential as a candidate kidney biomarker for HIVAN. Although the present study did not address the sensitivity or specificity of bFGF against proteinuria or microalbuminuria or other biomarkers for the early detection of HIVAN, bFGF could be used as an adjunctive marker for the detection of kidney disease in HIV infected children.

\section{Conclusion}

The present study measured significant differences in bFGF levels in children with HIVAN and control groups, although it failed to distinguish between HIVAN and idiopathic FSGS. The significant difference between bFGS in children with HIVAN compared to the HIV-positive and -negative control groups suggests that bFGF may be a useful biomarker for distinguishing HIVAN from HIVpositive children with no kidney disease. In HIV-infected children, one can potentially minimise the risk of developing kidney disease by beginning ART early in the course of the disease and continuing with the treatment. Studies with a larger sample size drawn from a wider range of ethnic groups are required to further assess bFGF as a potential biomarker for the detection of HIVAN in children. It is also essential to investigate other new biomarkers, to be used in concert with bFGF, as an approach to making a more conclusive diagnosis of HIVAN.

Declaration. This manuscript was submitted in partial fulfilment of the requirements for a MMedSci degree, awarded to NZG, at the University of KwaZulu-Natal (UKZN).

Acknowledgements. We appreciatively acknowledge the patients who participated in this study as well as the King Edward VIII and Inkosi Albert Luthuli hospitals' nurses for their assistance during the sample collection. We would also like to thank the Optics and Imaging Centre at the Doris Duke Medical Research Institute (DDMRI), Nelson Mandela School of Medicine, UKZN, for the use of the Bio-Plex system.

Author contributions. NZG collected the data, analysed results and wrote the manuscript. RB and TN analysed the data and edited the manuscript.

Funding. The authors would like to acknowledge financial support from the College of Health Sciences, UKZN, and the National Research Foundation for a grant (UID: 108132) awarded to RB.

Conflicts of interest. None.

1. Soler-García ÁA, Rakhmanina NY, Mattison PC, Ray PE. A urinary biomarker profile for children with HIV-associated renal diseases. Kidney Int 2009;76(2):207-214. https://doi.org/10.1038/ki.2009.115

2. Kalayjian RC. The treatment of HIV-associated nephropathy. Adv Chronic Kidney Dis 2010;17(1):59-71. https://doi.org/10.1053/j.ackd.2009.08.013

3. Bhimma R Purswani MU, Kala U. Kidney disease in children and adolescents with perinatal HIV-1 infection. J Int AIDS Soc 2013;16(1):18596. https://doi. org/10.7448/ias.16.1.18596

4. Wyatt CM, Klotman PE. HIV-associated nephropathy. In: Lifton RP, Somlo S, Giebisch GH, Seldin DW, eds. Genetic Diseases of the Kidney. London: Academic Press, 2009:793-813. https://doi.org/10.1016/B978-0-12-449851-8.00047-4.

5. Husain NE, Ahmed MH, Almobarak AO, et al. HIV-associated nephropathy in Africa: Pathology, clinical presentation and strategy for prevention. J Clin Med Res 2018;10(1):1-8. https://doi.org/10.14740/jocmr3235w

6. Roy S, Stapleton FB. Focal segmental glomerulosclerosis in children: Comparison of nonedematous and edematous patients. Pediatr Nephrol 1987;1(3):281-285. https://doi.org/10.1007/bf00849224

7. Kiffel J, Rahimzada Y, Trachtman H. Focal segmental glomerulosclerosis and chronic kidney disease in pediatric patients. Adv Chronic Kidney Dis 2011;18(5):332-338. https://doi.org/10.1053/j.ackd.2011.03.005

8. Röling J, Schmid H, Fischereder M, Draenert R, Goebel F. HIV-associated kidney diseases and highly active antiretroviral therapy-induced nephropathy. Clin Infect Dis 2006;42(10):1488-1495. https://doi.org/10.1086/503566

9. Soni SS, Ronco C, Katz N, Cruz DN. Early diagnosis of acute kidney injury: the promise of novel biomarkers. Blood Purif 2009;28(3):165-174. https://doi. org/10.1159/000227785

10. Ray PE, Liu X-H, Xu L, Rakusan T. Basic fibroblast growth factor in HIVassociated hemolytic uremic syndrome. Pediatr Neurol 1999;13(7):586-593. https://doi.org/10.1007/s004670050749

11. Kopp JB. BMP receptors in kidney. Kidney Int 2000;58(5):2237-2238. https://doi. org/10.1111/j.1523-1755.2000.00402.x

12. Kopp JB, Smith MW, Nelson GW, et al. MYH9 is a major-effect risk gene for focal segmental glomerulosclerosis. Nat Genet 2008;40(10):1175. http:doi/ 10.1038/ ng.226

13. Liu -H, Aigner A, Wellstein A, Ray PE. Up-regulation of a fibroblast growth factor binding protein in children with renal diseases. Kidney Int 2001;59(5):17171728. https://doi.org/10.1046/j.1523-1755.2001.0590051717.x

14. Olawumi $\mathrm{H}$,Olatunii $\mathrm{P}$. The value of serum albumin in pre-treatment assessment and monitoring of therapy in HIV/AIDS patients. HIV Med 2006;7(6):351-355

15. Villanueva S, Contreras F, Tapia A, et al. Basic fibroblast growth factor reduces functional and structural damage in chronic kidney disease. Am J Physiol 2013;306(4):F430-F41. https://doi.org/10.1152/ajprenal.00720.2012

16. Fredrick F, Francis JM, Ruggajo PJ, Maro EE. Renal abnormalities among HIV infected children at Muhimbili National Hospital (MNH) - Dar es Salaam, Tanzania. BMC Nephrol 2016;17(1):30. https://doi.org/10.1186/ s12882-016-0242-6

17. Portale AA, Wolf $\mathrm{M}$, Jüppner $\mathrm{H}$, et al. Disordered FGF23 and mineral metabolism in children with CKD. Clin J Am Soc Nephrol 2014;9(2):344-353. https://doi.org/10.2215/cjn.05840513

18. Atta MG, Gallant JE, Rahman MH, et al. Antiretroviral therapy in the treatment of HIV-associated nephropathy. Nephrol Dial Transplant 2006;21(10):28092813. https://doi.10.1093/ndt/gfl337 
19. Olawumi $\mathrm{H}$, Olatunji P. The value of s erum albumin in pretreatment assessment and monitoring of therapy in HIV/AIDS patients. HIV Med 2006;7(6):351355. https://doi.org/10.1111/j.1468-1293.2006.

20. Wyatt CM. Kidney disease and HIV infection. Top Antivir Med 2017;25(1):13.

21. Klahr S, Morrissey JJ. The role of vasoactive compounds, growth factors and cytokines in the progression of renal disease. Kidney Int 2000;57:S7-S14. https://doi.org/10.1046/j.1523-1755.57.s75.9.x
22. Tang P, Jerebtsova M, Przygodzki R, Ray PE. Fibroblast growth factor-2 increases the renal recruitment and attachment of HIV-infected mononuclear cells to kidney tubular epithelial cells. Pediatr Neurol 2005;20(12):1708-1716. https://doi.org/10.1007/s00467-005-2018-2

Accepted 31 July 2019 